\title{
Meta-analysis of individual and combined effects of mycotoxins on growing pigs
}

\author{
Ines Andretta ${ }^{\text {* }}$, Marcos Kipper², Luciano Hauschild ${ }^{3}$, Cheila Roberta Lehnen ${ }^{4}$, Aline Remus ${ }^{3}$, Raquel Melchior ${ }^{1}$
}

${ }^{1}$ Federal University of Rio Grande do Sul - Dept. of Animal Sciences, Av. Bento Gonçalves, 7712 - 91540-000 - Porto Alegre, RS - Brazil.

${ }^{2}$ Federal University of Santa Maria - Dept. of Animal Sciences, Av. Roraima, 1000 - 97105-900 - Santa Maria, RS - Brazil.

${ }^{3}$ São Paulo State University - Dept. of Animal Sciences, Via de Acesso Prof. Paulo Donato Castellane, s/n - 14884-900

- Jaboticabal, SP - Brazil.

${ }^{4}$ State University of Ponta Grossa - Dept. of Animal

Sciences, Av. General Carlos Cavalcanti, 4748 - 84030-900

- Ponta Grossa, PR - Brazil.

*Corresponding author <ines.andretta@ufrgs.br>

Edited by: Gerson Barreto Mourão

Received April 17, 2015

Accepted September 30, 2015
ABSTRACT: Little is known about the toxicity of concomitantly occurring mycotoxins in pig diets. This study was conducted to evaluate, through meta-analysis, the individual and the combined effects of mycotoxins on pig performance. The meta-analysis followed three sequential analyses (graphical, correlation, and variance-covariance) based on a database composed of 85 published papers, 1,012 treatments and 13,196 animals. Contamination of diets with individual mycotoxins reduced $(p<0.05)$ feed intake by $14 \%$ and weight gain by $17 \%$, while combined mycotoxins reduced the same responses by $42 \%$ and $45 \%$, respectively, in comparison with the non-challenged group. The correlation $(p<0.05)$ between reduction in weight gain $(\Delta G)$ and reduction in feed intake $(\Delta \mathrm{FI})$ was 0.67 in individual challenges and 0.93 in combined challenges. The estimated $\Delta \mathrm{G}$ was $-6 \%$ in individual challenges and $-7 \%$ in combined challenges when $\Delta \mathrm{Fl}$ was zero, suggesting an increase in the maintenance requirements of challenged animals. Most of $\Delta G$ (58\% in individual challenges and $84 \%$ in combined challenges) was attributed to the changes in feed efficiency. The association of mycotoxins enhances individual toxic effects and the $\Delta \mathrm{Fl}$ is important in explaining the deleterious effects on the growth of challenged pigs. Keywords: modelling, sanitary challenge, swine, toxicology

\section{Introduction}

Cereals are particularly susceptible to fungal contamination during both the pre- and postharvest stages. Many of these fungi are producers of mycotoxins, which are a chemically diverse group of secondary metabolites with a wide range of toxic effects (Bryden, 2012). The contamination of grain by mycotoxins represents a major concern worldwide in the pig production chain, since the effect of these toxins is to reduce animal performance (Andretta et al., 2012).

Several mycotoxins may be produced concomitantly in the same substrate. In addition, cross-contamination among raw ingredients in feed mills also contributes to increases in the incidence of combined mycotoxins in animal diets. Interactions between mycotoxins are complex and may result in the association of individual toxic properties or additive effects in the challenged animals (Speijers and Speijers, 2004). Safe tolerance levels may be also changed by interactions between toxins.

The diagnosis of mycotoxin co-contamination is difficult but essential to the expansion of knowledge in this important research area. Lower performance responses are expected for pigs challenged by combined mycotoxins compared with individual contamination, but few studies have quantified this effect and evaluated possible interference in maintenance requirements. This study aimed to evaluate and quantify, through meta-analysis, both the individual and combined effects of mycotoxins on the performance of growing pigs, and is a complementary approach to a previously published meta-analysis in which the challenge characteristic (individual/combined) was not assessed (Andretta et al., 2012).

\section{Materials and Methods}

Studies published in indexed journals reporting performance data of pigs challenged with mycotoxins were selected. The search through scientific papers was performed on digital databases. The main criteria for paper selection were: (a) presence of mycotoxins in diet; (b) a complete range covering nursery to finishing rearing phases; and (c) performance responses (feed intake and weight gain). After selection of the papers, the information relating to the proposed theoretical model and other additional variables were copied from the material and methods and the results sections in the original publication, and transferred to an electronic spreadsheet.

The methodology adopted for defining both the dependent and independent variables and for coding the data followed the proposals described in the literature (Sauvant et al., 2008). Codes, based on qualitative grouping criteria in the analytical models, were used for detecting the presence of challenge (control or contaminated diet) and type of contamination (control, diet contaminated with a single mycotoxin, or diet contaminated with a combination of mycotoxins). Other codes were used as moderating variables in the analysis for the consideration of variability in the experiments conducted (article, inter-, and intra-effects).

The variables analyzed were experimental characteristics and performance responses (feed intake, weight 
gain, and calculated feed conversion ratio). The metaanalysis followed three sequential analyses: graphic (to evaluate the quality of the database and to verify the biological consistency of the data), correlation (to identify possible relationships between the different variables), and variance-covariance (to compare the groups and to generate the prediction equations).

The equations obtained by variance-covariance analysis were used to study the relationship between feed intake and weight gain of challenged pigs. For this purpose, the performance responses of challenged treatments were relativized to the respective control (nonchallenged) treatment and expressed in terms of percentage in variation. This procedure was adopted because it considerably reduces the effect of the variation between experiments in the database. Relative data were used to generate equations that estimated the relationship between the variation in weight gain $(\Delta G)$ and the variation in feed intake $(\Delta \mathrm{FI})$. Equations were fitted independently for animals challenged by a single mycotoxin or a combination of mycotoxins. The relationship was considered quadratic as all components of the equations were significant $(p<0.05)$ in this analysis. The variation between the experiments in the database was considered in the models as a fixed effect. The intercepts of the equations were empirically associated with the maintenance requirements (reduction in weight gain not related to the reduction in feed intake), while the slopes were interpreted as an indication of changes associated with feed efficiency (reduction in weight gain associated with the reduction in feed intake). The partition between these two effects was calculated using (1) the adjusted $\Delta \mathrm{G}$ mean (obtained from previously fitted equations) and (2) the $\Delta$ FI mean observed in the database. A similar interpretation has been used in previous studies on health challenges (Pastorelli et al., 2012; Remus et al., 2014).

The effect of dietary concentration of mycotoxins on health challenges was also studied using prediction equations. Relative performance data $(\Delta \mathrm{FI}$ and $\Delta \mathrm{G})$ and dietary mycotoxin doses (considering the sum of concentrations when treatments used combined mycotoxins) were used. All analyses were performed using Minitab statistical software (Minitab for Windows, version 16).

\section{Description of the database}

The database was composed of 85 papers published between 1968 and 2010 (mode year: 2002). The studies comprised a total of 13,196 pigs, with an average of 17 animals per treatment. Average initial and final ages were 44 days (ranging between 21 and 160 days) and 68 days (27-224 days), respectively. Nursery piglets were used in $64 \%$ of the experiments. The growing phase was studied in $18 \%$ of the articles, the finishing phase in $3 \%$, and total rearing period (growing and finishing) in $15 \%$.

In total, 1,011 treatments were considered in the analyses, out of which $35 \%$ were classified as control treatments. Considering the challenged groups, $86 \%$ used individual mycotoxins and $14 \%$ combined mycotoxins. The average dietary concentration of mycotoxins were $0.485 \mathrm{ppm}$ for aflatoxins (maximum of 4 ppm), 3.63 for deoxynivalenol (maximum of $72 \mathrm{ppm}$ ), $1.14 \mathrm{ppm}$ for zearalenone (maximum of $9 \mathrm{ppm}$ ), and $23.2 \mathrm{ppm}$ for fumonisins (maximum of $120 \mathrm{ppm}$ ). Most experiments with combinations of mycotoxins evaluated groups of mycotoxins produced by the same fungus genus (for example, Fusarium toxins). Corn and soybean meal were the main feedstuffs in $43 \%$ of the experimental diets.

\section{Results and Discussion}

Mycotoxins affected the performance responses of pigs (Table 1). The dietary exposure to a single mycotoxin reduced feed intake by $14 \%(p<0.05)$ relative to the control treatment, while combined mycotoxins reduced the same response by $42 \%$. Feed intake means adjusted for body weight were also lower $(p<0.05)$ in pigs fed diets containing combined mycotoxins relative to those fed individual mycotoxins.

Weight gain was $17 \%$ lower $(p<0.05)$ in pigs challenged by individual mycotoxins and $45 \%$ lower $(p<0.05)$ in those challenged by combined mycotoxins relative to the control treatment. Weight gain adjusted for body weight was $22 \%$ lower $(p<0.05)$ in pigs challenged by combined mycotoxins relative to those challenged by single toxins.

Feed conversion ratio was higher $(p<0.05)$ in pigs exposed to mycotoxins relative to non-challenged animals. However, groups challenged by individual and combined mycotoxins showed a similar $(p>0.05)$ feed conversion ratio, possibly because feed intake and weight gain responses were influenced by mycotoxins to a similar magnitude within each challenge type.

The effect of combined mycotoxins is complex and may be classified into synergic, additive, and antagonist categories. A number of limitations in experimental designs impeded the identification and classification of the

Table 1 - Performance responses obtained by meta-analysis, of growing pigs fed diets containing mycotoxins in individual or combined challenges.

\begin{tabular}{|c|c|c|c|c|c|}
\hline & \multicolumn{3}{|c|}{ Treatments } & \multirow{3}{*}{$p^{1}$} & \multirow{3}{*}{$\mathrm{RSD}^{2}$} \\
\hline & \multirow{2}{*}{ Control } & \multicolumn{2}{|c|}{ Mycotoxins } & & \\
\hline & & Individual & Combined & & \\
\hline Daily feed intake (FI), kg & $1.48^{\mathrm{a}}$ & $1.27^{\mathrm{b}}$ & $0.86^{c}$ & *** & 0.07 \\
\hline Adjusted $\mathrm{Fl}^{3}, \mathrm{~kg}$ & $1.32^{\mathrm{a}}$ & $1.19^{\mathrm{b}}$ & $1.14^{c}$ & $* *$ & 0.04 \\
\hline Daily weight gain (G), kg & $0.64^{\mathrm{a}}$ & $0.53^{b}$ & $0.35^{c}$ & *** & 0.05 \\
\hline Adjusted $\mathrm{G}^{3}, \mathrm{~kg}$ & $0.58^{\mathrm{a}}$ & $0.50^{\mathrm{b}}$ & $0.45^{c}$ & * & 0.03 \\
\hline Feed conversion ratio (FCR) & $2.32^{\mathrm{a}}$ & $2.66^{b}$ & $2.75^{\mathrm{b}}$ & * & 0.08 \\
\hline Adjusted FCR 3 & $2.18^{a}$ & $2.49^{b}$ & $2.42^{b}$ & ${ }^{* *}$ & 0.12 \\
\hline
\end{tabular}

Probability indicated by ${ }^{*},{ }^{* *}$ or ${ }^{* * *}$ significant at $0.05,0.01$ or 0.001 levels respectively; ${ }^{2}$ Residual standard deviation; ${ }^{3}$ Values adjusted by covariance to average live body weight; Means followed by different letters within a line are significantly different $(p<0.05$, Tukey test). 
effects of combined mycotoxins in all studies included in the database. Only studies developed using factorial design allowed for these assessments. It was possible to observe synergic (Dilkin et al., 2003) and additive (Harvey et al., 1991) interactions between co-occurring mycotoxins in body weight gain. An additive effect was also reported for feed intake response of challenged pigs (Harvey et al., 1991). Although antagonists of co-occurring mycotoxins effects were not observed for performance responses in the database studies, previously published meta-analysis reported this effect in several serum parameters of challenged pigs (Grenier and Oswald, 2011).

The correlation between $\Delta \mathrm{G}$ and $\Delta \mathrm{FI}$ was lower in challenges by individual mycotoxins $(\mathrm{r}=0.67 ; p<$ $0.05)$ compared to challenges by combined mycotoxins $(\mathrm{r}=0.97 ; p<0.05) . \Delta \mathrm{G}$ showed a quadratic relationship with $\Delta$ FI (Figure 1). The intercepts of the equations were different from zero and negative in both challenges. According to the equations, pigs fed diet containing a single mycotoxin presented a $\Delta \mathrm{G}$ of $-6 \%$ when $\Delta \mathrm{FI}$ is null. Under the same conditions, pigs challenged by combined mycotoxins showed a $\Delta \mathrm{G}$ of $-7 \%$. These values may indicate an increase in maintenance requirements of challenged pigs, as previously described by Pastorelli et al., (2012) for mycotoxicoses and other health challenges. It may also be inferred that the magnitude of this effect on the maintenance requirements is little influenced by how the mycotoxins are presented in the diets, because the intercepts were numerically similar in the equations for both individual and combined challenges.

The partition of the effects on $\Delta \mathrm{G}$ corrected for the average $\Delta \mathrm{FI}$ is presented in Figure 2 . In both challenges, most of $\Delta \mathrm{G}$ (58\% in individual challenges and $84 \%$ in the combined challenges) was related to changes in feed efficiency. Although other pathogenesis mechanisms should not be disregarded, current results highlight the

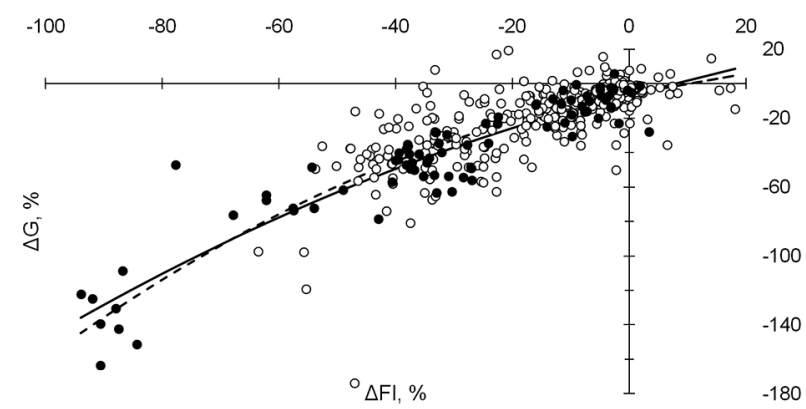

Figure 1 - Relationship between weight gain variation $(\Delta \mathrm{G}$, comparison between control and challenged pigs) and the feed intake variation $(\Delta \mathrm{Fl})$, obtained by meta-analysis, of growing pigs fed diets containing mycotoxins in individual ${ }^{1}$ or combined ${ }^{2}$ challenges. ${ }^{1}$ Observed values represented by white circles (O) and equation $\left(y=-6.089+0.6015 x-0.0093 x^{2} ; R^{2}=0.65\right)$ represented by dotted line $(--)$; ${ }^{2}$ Observed values represented by black circles $(\bullet)$ and equation $\left(y=-6.559+0.8438 x-0.0057 x^{2} ; R^{2}=0.87\right)$ represented by a continuous line (-). importance of better understanding the mechanisms by which the mycotoxins interfere with the animals' feeding behavior.

Some theories assert that alimentary toxins may act as limiting factors in diet selection, because animals relate their consumption to the metabolic consequences of discomfort or specific clinical signs (Hedman et al., 1997). Diets produced with naturally-contaminated grains may also be refused by the animals due to the organoleptic changes caused by fungal contaminants (Akande et al., 2006). In general, the mechanism related to feed intake reduction in challenged pigs varies according to the mycotoxins (or their combinations) present in the diet. Although specific effects were expected for each mycotoxin, it was not possible to perform individual analyses due to the database characteristics.

Changes in protein synthesis and enzyme kinetics are usually described as mechanisms by which the mycotoxins may interfere in pig performance (Marin et al., 2002; Walle et al., 2010). In the present study, most of the mycotoxin effect on weight gain was explained by changes in feed efficiency. Thus, the effects of mycotoxins on pig growth may be strongly associated with primary modes of action, such as reduced feed intake (Andretta et al., 2012). In this context, it is possible to infer that some feeding and nutritional practices could be evaluated as alternatives for alleviating the effects of mycotoxin on pig growth.

Dietary concentration of mycotoxins is an important factor when considering intoxication (Andretta et al., 2012). The equations estimating the $\Delta \mathrm{FI}$ and $\Delta \mathrm{G}$ of challenged pigs according to the dietary concentration of mycotoxins are presented in Table 2 . The equations considered the sum of individual mycotoxin concentrations when treatments used combined mycotoxins. It was not possible to analyze concentrations of specific mycotoxins

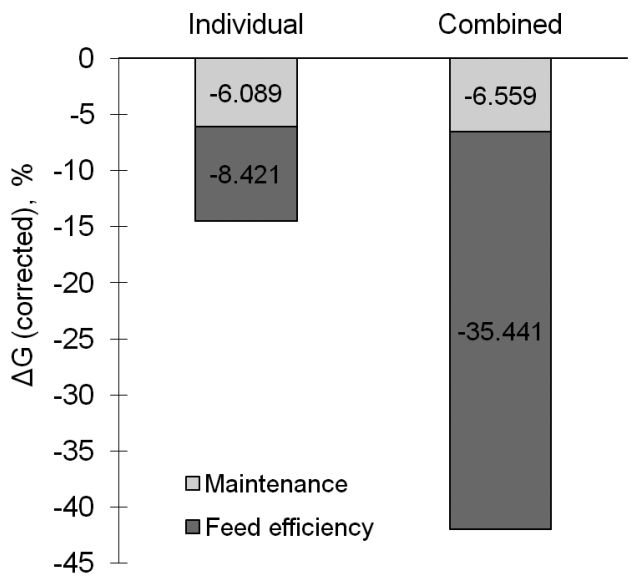

Figure 2 - Partitioning of the weight gain variation (corrected $\Delta G$, comparison between control and challenged pigs) between the fraction due to the change in maintenance requirement or due to the change in feed efficiency of growing pigs fed diets containing mycotoxins in individual or combined challenges. 
Table 2 - Equations estimating the variation in feed intake $(\Delta \mathrm{Fl}$, comparison between control and challenged pigs, expressed as $\%)$ and in weight gain $(\Delta \mathrm{G}, \%)$ of growing pigs fed diets containing mycotoxins in individual or combined challenges, according to the dietary concentration of mycotoxins ( $x$, expressed in ppm).

\begin{tabular}{llcc}
\hline Response $(\mathrm{y})$ & Challenge & Equation & $\mathrm{R}^{2}$ \\
\hline$\Delta \mathrm{FI}$ & Individual & $-12.62+0.1139 x$ & 0.46 \\
$\Delta \mathrm{FI}$ & Combined & $-29.25+0.3338 \mathrm{x}$ & 0.64 \\
$\Delta \mathrm{G}$ & Individual & $-18.45+0.1833 \mathrm{x}$ & 0.57 \\
$\Delta \mathrm{G}$ & Combined & $-33.86+0.5313 \mathrm{x}$ & 0.61 \\
\hline
\end{tabular}

due to the diversity of combinations tested in the database. Despite not taking into account the variation between mycotoxins, the equations evidenced the stronger effect of mycotoxins when co-occurring in the feed. The intercepts of the equations for both responses $(\Delta \mathrm{FI}$ and $\Delta \mathrm{G})$ were higher when pigs were challenged with combined mycotoxins compared to individual contamination. Also supportive of this hypothesis was the fact that the slopes obtained for the effect of combined mycotoxins were $193 \%$ higher for the $\Delta \mathrm{FI}$ and $190 \%$ higher for the $\Delta \mathrm{G}$ compared to those found for individual challenges. Thus, it is possible to infer that the same mycotoxin concentration showed a stronger effect on pig performance when present in combination with other mycotoxins than in a single contamination.

The toxicity and the clinical signs observed when more than one mycotoxin is ingested are complex and diverse (Huff et al., 1988). Our results indicate that the association of mycotoxins enhances isolated toxic effects of these substances. Thus, the identification of key critical control points in the cereal production chain is strongly recommended to prevent the co-occurrence of mycotoxins in feeds and, thus, their negative effects on pig production.

\section{Acknowledgments}

To the São Paulo Research Foundation (FAPESP, Grant $\mathrm{n}^{\circ}$. 2012/03781-0) and the Coordination for the Improvement of Higher Level Personnel (CAPES) for financial support.

\section{References}

Akande, K.E.; Abubakar, M.M.; Adegbola, T.A.; Bogoro, S.E. 2006. Nutritional and health implications of mycotoxins in animal feeds: a review. Pakistan Journal of Nutrition 5: 398-403.
Andretta, I.; Kipper, M.; Lehnen, C.R.; Hauschild, L.; Vale, M.M.; Lovatto, P.A. 2012. Meta-analytical study of productive and nutritional interactions of mycotoxins in growing pigs. Animal 6: $1476-1482$.

Bryden, W. 2012. Mycotoxin contamination of the feed supply chain: implications for animal productivity and feed security. Animal Feed Science and Technology 173: 134-158.

Dilkin, P.; Zorzete, P.; Mallmann, C.A.; Gomes, J.D.; Utiyama, C.E.; Oetting, L.L.; Corrêa, B. 2003. Toxicological effects of chronic low doses of aflatoxin $\mathrm{B}(1)$ and fumonisin $\mathrm{B}(1)$ containing Fusarium moniliforme culture material in weaned piglets. Food and Chemical Toxicology 41: 1345-1353.

Harvey, R.B.; Kubena, L.F.; Elissalde, M.H.; Corrier, D.E.; Huff, W.E.; Rottinghaus, G.E.; Clement, B.A. 1991. Cocontamination of swine diets by aflatoxin and diacetoxyscirpenol. Journal of Veterinary Diagnostic Investigation 3: 155-160.

Hedman, R.; Thuvander, A.; Gadhasson, I.; Reverter, M.; Pettersson, H. 1997. Influence of dietary nivalenol exposure on gross pathology and selected immunological parameters in young pigs. Natural Toxins 5: 238-246.

Huff, W.E.; Kubena, L.F.; Harvey, R.B.; Doerr, J.A. 1988. Mycotoxin interactions in poultry and swine. Journal of Animal Science 66: 2351-2355.

Grenier, B.; Oswald, I.P. 2011. Mycotoxin co-contamination of food and feed: meta-analysis of publications describing toxicological interactions. World Mycotoxin Journal 4: 285-313.

Marin, D.E.; Taranu, I.; Bunaciu, R.P.; Pascale, F.; Tudor, D.S.; Avram, N.; Sarca, M.; Cureu, I.; Criste, R.D.; Suta, V.; Oswald, I.P. 2002. Changes in performance, blood parameters, humoral and cellular immune responses in weanling piglets exposed to low doses of aflatoxin. Journal of Animal Science 80: 12501257.

Pastorelli, H.; Milgen, J.; Lovatto, P.; Montagne, L. 2012. Metaanalysis of feed intake and growth responses of growing pigs after a sanitary challenge. Animal 6: 952-961.

Remus, A.; Hauschild, L.; Andretta, I.; Kipper, M.; Lehnen, C.R.; Sakomura, N.K. 2014. A meta-analysis of the feed intake and growth performance of broiler chickens challenged by bacteria. Poultry Science 93: 1149-1158.

Sauvant, D.; Schmidely, P.; Daudin, J.J.; St-Pierre, N.R. 2008. Meta-analyses of experimental data in animal nutrition. Animal 2: 1203-1214.

Speijers, G.J.A.; Speijers, M.H.M. 2004. Combined toxic effects of mycotoxins. Toxicology Letters 153: 91-98.

Walle, J.V. van de; Sergent, T.; Piront, N.; Toussaint, O.; Schneider, Y.J.; Larondelle, Y. 2010. Deoxynivalenol affects in vitro intestinal epithelial cell barrier integrity through inhibition of protein synthesis. Toxicology and Applied Pharmacology 245: 291-298. 